\title{
Erratum: Hereditary terms at next-to-leading order in two-body gravitational dynamics [Phys. Rev. D 101, 064033 (2020)]
}

\author{
Stefano Foffa $\odot$ and Riccardo Sturani॰
}

(Received 5 March 2021; published 12 April 2021)

DOI: 10.1103/PhysRevD.103.089901

It is incorrectly said that in Sec. II that $A_{\text {her }}$ defined in Eq. (5) involves Feynman propagators. For the memory integral studied in Sec. IIIB. 5 one has actually to consider retarded (advanced) propagators for the $k, q(p)$ terms, see [1] for a discussion of Green's function boundary condition. As an additional consequence, the expression in Eq. (25) is incorrect as it has to be symmetrized (averaged) over its argument $\left(k_{0}, \mathbf{k}\right),\left(q_{0}, \mathbf{q}\right),\left(p_{0}, \mathbf{p}\right)$, with $p \equiv-k-q$, its correct version being

$$
\begin{aligned}
i S_{\mathrm{eff} 5 \mathrm{PN}}^{Q^{3}}= & -i 128 \pi^{2} G_{d}^{2} \int \frac{\mathrm{d} q_{0}}{2 \pi} \frac{\mathrm{d} k_{0}}{2 \pi} Q_{i j}\left(k_{0}\right) Q_{m n}\left(q_{0}\right) Q_{k l}\left(p_{0}\right) \\
& \times \int_{\mathbf{k}, \mathbf{q}} \frac{1}{\mathbf{k}^{2}-k_{0}^{2}} \frac{1}{\mathbf{p}^{2}-p_{0}^{2}} \frac{1}{\mathbf{q}^{2}-q_{0}^{2}} \frac{1}{3}\left[V^{i j m n k l}(k, q, p)+V^{i j m n k l}(p, k, q)+V^{i j m n k l}(q, p, k)\right],
\end{aligned}
$$

with

$$
\begin{array}{rlrl}
V^{i j m n k l}(k, q, p)= & \left\{\frac{p_{0}^{2} k_{0}^{2} q_{0}^{2}}{8} \delta_{i m} \delta_{j k} \delta_{l n}\left(-k_{0} q_{0}+\mathbf{k} \cdot \mathbf{q}\right)\right. & \left\{\sigma^{3}\right\} \\
& -\frac{p_{0}^{3} q_{0} k_{0}^{2}}{4} q_{n} \delta_{j l}\left(k_{k} \delta_{i m}-k_{i} \delta_{k m}\right) & \left\{\sigma^{2} A\right\} \\
& +0 & \left\{\sigma^{2} \phi\right\} \\
& -\frac{1}{4} \delta^{j l} p_{0}^{2} k_{0} k_{i} q_{m} q_{n}\left(q_{0} k_{k}-k_{0} q_{k}\right) & \{\sigma A \phi\} \\
& +\frac{1}{8} p_{0}^{2} k_{0} q_{0} k_{i} q_{m}\left[\left(\mathbf{k} \cdot \mathbf{q}-k_{0} q_{0}\right) \delta_{n k} \delta_{j l}+k_{l} q_{k} \delta_{n j}\right. & \\
& \left.+\delta_{n l}\left(k_{j} q_{k}-k_{k} q_{j}\right)+\delta_{j k}\left(k_{l} q_{n}-k_{n} q_{l}\right)\right] & & \left\{\sigma A^{2}\right\} \\
& -\frac{1}{8 c_{d}} p_{0}^{2} k_{i} k_{j} k_{k} q_{l} q_{m} q_{n} & & \left\{\sigma \phi^{2}\right\} \\
& -\frac{1}{4} p_{0}^{2} k_{0} q_{0} k_{i} p_{k} q_{m}\left(q_{j} \delta_{n l}-q_{l} \delta_{n j}+q_{n} \delta_{l j}\right) & & \left\{A^{3}\right\} \\
& +\frac{1}{8} k_{0} p_{0} k_{i} p_{k} q_{m} q_{n}\left(\mathbf{k} \cdot \mathbf{p} \delta_{j l}+k_{j} p_{l}-k_{l} p_{j}\right) & & \left\{A^{2} \phi\right\} \\
& +\frac{1}{4 c_{d}} k_{0} p_{0} k_{i} k_{j} p_{k} q_{l} q_{m} q_{n} & & \left\{A \phi^{2}\right\} \\
& \left.-\frac{1}{8 c_{d}} k_{0} p_{0} k_{i} k_{j} p_{k} p_{l} q_{m} q_{n}\right\} & \left\{\phi^{3}\right\} .
\end{array}
$$

As a consequence, the numerical factors in Eqs. (26) and (27) are incorrect, the correct ones being, respectively,

$$
S_{\text {eff5PN }}^{Q^{3}}=-\frac{G_{N}^{2}}{15} \int \mathrm{d} t\left[\dddot{Q}_{i l} \dddot{Q}_{j l} Q_{i j}+\frac{4}{7} \dddot{Q}_{i l} \dddot{Q}_{j l} \ddot{Q}_{i j}\right]
$$

and 


$$
\begin{aligned}
S_{\mathrm{eff} 5 \mathrm{PN}}^{(\mathrm{far})}= & G_{N}^{2} \int \mathrm{d} t\left[M\left(\frac{82}{6615} \dddot{O}_{i j k}^{2}+\frac{508}{675} \dddot{J}_{i j}^{2}\right)\right. \\
& \left.+\frac{8}{15} \dddot{Q}_{i l} \dddot{Q}_{j l} \epsilon_{i j k} L_{k}-\frac{1}{15} Q_{i l} \dddot{Q}_{j l} \dddot{Q}_{i j}-\frac{4}{105} \dddot{Q}_{i l} \dddot{Q}_{j l} \ddot{Q}_{i j}\right]
\end{aligned}
$$

Conclusions are otherwise unchanged.

[1] S. Foffa and R. Sturani, arXiv:2103.03190. 